\title{
ON SOME NEW PROPERTIES OF THE SPHERICAL CURVATURE OF STEREOGRAPHICALLY PROJECTED ANALYTIC CURVES
}

\author{
STEPHEN M. ZEMYAN
}

Received 2 August 2002

\begin{abstract}
We discover new information about the spherical curvature of stereographically projected analytic curves. To do so, we first state formulas for the spherical curvature and spherical torsion of the curves on $S^{2}$ which result after stereographically projecting the image curves of analytic, univalent functions belonging to the class $\mathscr{9}$. We then derive results concerning the location of the critical points of the spherical curvature, considered both as a function of one and two variables. Further analysis leads to a maximum principle for the spherical curvature functions.
\end{abstract}

2000 Mathematics Subject Classification: 30C55.

1. Introduction. We begin by defining the spherical curvature and spherical torsion of the stereographically projected images of circles and radii in $U$.

Let $\mathscr{S}$ denote the class of analytic, univalent functions $f(z)$ defined in the unit disk $U=\{z:|z|<1\}$ and normalized so that $f(0)=0$ and $f^{\prime}(0)=1$.

Let $f \in \mathscr{S}$ and let $\Pi$ denote the stereographic projection of the image plane of $f$ onto the unit sphere $S^{2}$. For each fixed $r, 0<r<1$, let

$$
\mathscr{C}_{r}=\{z:|z|=r\}, \quad \mathscr{C}_{r}^{\prime}=f\left(\mathscr{C}_{r}\right), \quad \mathscr{C}_{r}^{\prime \prime}=\Pi\left(\mathscr{C}_{r}^{\prime}\right) .
$$

For each fixed $\theta,-\pi<\theta \leq+\pi$, let

$$
\mathscr{L}_{\theta}=\{z: \arg z=\theta\}, \quad \mathscr{L}_{\theta}^{\prime}=f\left(\mathscr{L}_{\theta}\right), \quad \mathscr{L}_{\theta}^{\prime \prime}=\Pi\left(\mathscr{L}_{\theta}^{\prime}\right) .
$$

The local curvature at a specified point on each of the curves $\mathscr{C}_{r}^{\prime}$ and $\mathscr{L}_{\theta}^{\prime}$ has been the object of intense research [1, pages 126 and 262]. Of course, the local torsion at any specified point on each of these curves is equal to zero. Our immediate objective is to determine formulas for the spherical curvature and spherical torsion at a specified point on the stereographically projected curves $\mathscr{C}_{r}^{\prime \prime}$ and $\mathscr{L}_{\theta}^{\prime \prime}$ on the unit sphere $S^{2}$.

A parametrization of the curves under consideration is easily prescribed. Indeed, if we write

$$
f(z)=f(r, \theta)=(u(r, \theta), v(r, \theta)) \quad\left(z=r e^{i \theta} \in U\right),
$$


then

$$
\mathscr{C}_{r}^{\prime \prime}=\{X(r, \theta):-\pi<\theta \leq+\pi\}, \quad \mathscr{L}_{\theta}^{\prime \prime}=\{X(r, \theta): 0<r<1\},
$$

where

$$
X(r, \theta)=\left(\frac{2 u(r, \theta)}{|f|^{2}+1}, \frac{2 v(r, \theta)}{|f|^{2}+1}, \frac{|f|^{2}-1}{|f|^{2}+1}\right)
$$

In terms of the parametrization (1.5), the local spherical curvature $\kappa(\theta ; r, f)$ and the local spherical torsion $\tau(\theta ; r, f)$ at the point $X(r, \theta)$ on the curve $\mathscr{C}_{r}^{\prime \prime}$ are classically defined by the formulas

$$
\kappa(\theta ; r, f)=\frac{\left|X_{\theta} \times X_{\theta \theta}\right|}{\left|X_{\theta}\right|^{3}}, \quad \tau(\theta ; r, f)=\frac{\left[X_{\theta} X_{\theta \theta} X_{\theta \theta \theta}\right]}{\left|X_{\theta} \times X_{\theta \theta}\right|^{2}},
$$

where the subscripts on $X$ denote the variable with respect to which the partial derivative is taken. The local spherical curvature $\kappa(r ; \theta, f)$ and spherical torsion $\tau(r ; \theta, f)$ at the point $X(r, \theta)$ on the curve $\mathscr{L}_{\theta}^{\prime \prime}$ are given by similar formulas.

In our first result below, we provide explicit formulas for the spherical curvatures and spherical torsions under study. In all results to follow, the quantity

$$
f^{\#}(z)=\frac{\left|f^{\prime}(z)\right|}{1+|f(z)|^{2}}
$$

will denote the spherical derivative of $f(z)$ and the quantity

$$
\{f, z\}=\left(\frac{f^{\prime \prime}(z)}{f^{\prime}(z)}\right)^{\prime}-\frac{1}{2}\left(\frac{f^{\prime \prime}(z)}{f^{\prime}(z)}\right)^{2}
$$

will denote the Schwarzian derivative of $f(z)$. Note that (1.11) provides an explicit connection between these two quantities.

In all formulas to follow, the subscripts on $\mathscr{E}$ denote the variable(s) with respect to which the partial derivative is taken.

THEOREM 1.1. Let $f \in \mathscr{S}$ and let $\mathscr{L}(r, \theta ; f)=1 / r f^{\sharp}(r, \theta)$.

(a) At the point $X(r, \theta)$ on the sphere curve $\mathscr{C}_{r}^{\prime \prime}$, the local spherical curvature $\kappa(\theta ; r, f)$ is given by the formula

$$
\kappa(\theta ; r, f)=\left(1+\frac{1}{4} r^{2} \mathscr{E}_{r}^{2}(r, \theta ; f)\right)^{1 / 2},
$$

and the local spherical torsion $\tau(\theta ; r, f)$ is given by the formula

$$
\tau(\theta ; r, f)=\frac{r \mathscr{L}(r, \theta ; f) \mathscr{E}_{r \theta}(r, \theta ; f)}{4 \kappa^{2}(\theta ; r, f)} .
$$


Furthermore, since

$$
r \mathscr{L}_{r \theta}(r, \theta ; f)=\mathscr{L}(r, \theta ; f) \operatorname{Im}\left[z^{2}\{f, z\}\right] \quad\left(z=r e^{i \theta} \in U\right),
$$

these quantities are related by

$$
4 \kappa^{2}(\theta ; r, f) \tau(\theta ; r, f)=\mathscr{L}^{2}(r, \theta ; f) \operatorname{Im}\left[z^{2}\{f, z\}\right] .
$$

(b) At the point $X(r, \theta)$ on the sphere curve $\mathscr{L}_{\theta}^{\prime \prime}$, the local spherical curvature $\kappa(r ; \theta, f)$ is given by the formula

$$
\kappa(r ; \theta, f)=\left(1+\frac{1}{4} \mathscr{L}_{\theta}^{2}(r, \theta ; f)\right)^{1 / 2},
$$

and the local spherical torsion $\tau(r ; \theta, f)$ is given by the formula

$$
\tau(r ; \theta, f)=-\frac{r \mathscr{E}(r, \theta ; f) \mathscr{E}_{r \theta}(r, \theta ; f)}{4 \kappa^{2}(r ; \theta, f)} .
$$

Furthermore, these quantities are related by

$$
4 \kappa^{2}(r ; \theta, f) \tau(r ; \theta, f)=-\mathscr{L}^{2}(r, \theta ; f) \operatorname{Im}\left[z^{2}\{f, z\}\right] .
$$

The proof of this result is lengthy, but straightforward, and is omitted.

REMARK 1.2. In deriving formulas (1.9) and (1.13), it becomes clear that the partial derivatives $-r \mathscr{L}_{r}(r, \theta ; f)$ and $\mathscr{L}_{\theta}(r, \theta ; f)$ are actually the real and imaginary parts of the same quantity. Indeed, if we set

$$
\Phi(z ; f)=\frac{\left(1+z f^{\prime \prime}(z) / f^{\prime}(z)\right)-\left(2|f(z)|^{2} /\left(1+|f(z)|^{2}\right)\right)\left(z f^{\prime}(z) / f(z)\right)}{2 r f^{\sharp}(z)},
$$

then, with $z=r e^{i \theta}$, we have

$$
\begin{aligned}
& -\frac{1}{2} r \mathscr{L}_{r}(r, \theta ; f)=\operatorname{Re}\{\Phi(z ; f)\}, \\
& +\frac{1}{2} \mathscr{L}_{\theta}(r, \theta ; f)=\operatorname{Im}\{\Phi(z ; f)\} \text {. }
\end{aligned}
$$

Hence, the spherical curvatures are related to each other via $\Phi(z ; f)$.

REMARK 1.3. Since $f^{\prime}(z) \neq 0$ in $U$, the representation

$$
\mathscr{L}(r, \theta ; f)=\left|\frac{1}{z f^{\prime}(z)}\right|+\left|\frac{f^{2}(z)}{z f^{\prime}(z)}\right|
$$


shows that $\mathscr{E}(r, \theta ; f)$ is subharmonic in the punctured disk $U-\{0\}$. Indeed, with the additional formulas (to be used in the proof of Theorem 3.1)

$$
\begin{aligned}
\mathscr{L}_{r r}(r, \theta ; f)= & -\frac{1}{r} \mathscr{L}_{r}(r, \theta ; f)+\frac{2}{r^{2} \mathscr{L}(r, \theta ; f)}\left(1+|\Phi(z ; f)|^{2}\right) \\
& +\frac{\mathscr{L}(r, \theta ; f)}{r^{2}}\left[\frac{1}{2}-\operatorname{Re}\left[z^{2}\{f, z\}\right],\right. \\
\mathscr{L}_{\theta \theta}(r, \theta ; f)= & \frac{2}{\mathscr{L}(r, \theta ; f)}\left(1+|\Phi(z ; f)|^{2}\right) \\
& +\mathscr{L}(r, \theta ; f)\left[\operatorname{Re}\left[z^{2}\{f, z\}\right]-\frac{1}{2}\right],
\end{aligned}
$$

we can explicitly compute the Laplacian of $\mathscr{L}(r, \theta ; f)$ to be

$$
\Delta \mathscr{E}(r, \theta ; f)=\frac{4}{r^{2} \mathscr{L}(r, \theta ; f)}\left(1+|\Phi(z ; f)|^{2}\right)>0 .
$$

More generally, we see that $\mathscr{L}^{\alpha}(r, \theta ; f)$ is also subharmonic for every $\alpha>0$ since

$$
\Delta_{\mathscr{L}}^{\alpha}(r, \theta ; f)=\frac{4 \alpha}{r^{2} \mathscr{L}^{2-\alpha}(r, \theta ; f)}\left(1+\alpha|\Phi(z ; f)|^{2}\right)>0
$$

Also, we note that

$$
\begin{gathered}
\mathscr{L}_{r r}(r, \theta ; f)+\frac{1}{r} \mathscr{L}_{r}(r, \theta ; f)-\frac{1}{r^{2}} \mathscr{L}_{\theta \theta}(r, \theta ; f) \\
=\frac{2 \mathscr{L}(r, \theta ; f)}{r^{2}}\left[\frac{1}{2}-\operatorname{Re}\left[z^{2}\{f, z\}\right]\right] .
\end{gathered}
$$

In Section 2, for an arbitrary $f \in \mathscr{Y}$, we are able to determine all critical points of $\kappa(\theta ; r, f)$ and $\kappa(r ; \theta, f)$ when they are considered as functions of one variable, necessarily introducing the level sets $\lambda_{0}^{r}(f), \lambda_{0}^{\theta}(f)$, and $\Lambda_{0}(f)$. Using these data, a strategy for locating the extreme values of the spherical curvature $\kappa(\theta ; r, f)$ on the curve $\mathscr{C}_{r}^{\prime \prime}$ and the spherical curvature $\kappa(r ; \theta, f)$ on the curve $\mathscr{L}_{\theta}^{\prime \prime}$ is discussed.

In Section 3, for an arbitrary $f \in \mathscr{Y}$, we are also able to determine the stationary points of $\kappa(\theta ; r, f)$ and $\kappa(r ; \theta, f)$ when they are considered as functions of two variables.

To complement this result, in Section 4 , we show that on certain subdomains $D$, there exist real $\alpha$ and $\beta$ for which $\kappa^{\alpha}(\theta ; r, f)$ and $\kappa^{\beta}(r ; \theta, f)$ are subharmonic on $D$. Although it is not necessarily true that $\kappa(\theta ; r, f)$ and $\kappa(r ; \theta, f)$ are subharmonic, we will show nevertheless that maximum principles for these spherical curvature functions are still valid on certain subdomains of $U$.

Finally, in Section 5, we describe some topological properties of the level sets $\lambda_{0}^{r}(f), \lambda_{0}^{\theta}(f)$, and $\Lambda_{0}(f)$ which arise naturally in Sections 2,3 , and 4 . 
2. Critical points of the spherical curvature functions considered as functions of one variable. We now consider the problem of determining the critical points of the spherical curvature functions on the curves $\mathscr{C}_{r}^{\prime \prime}$ and $\mathscr{L}_{\theta}^{\prime \prime}$ for an arbitrary function $f \in \mathscr{Y}$. This is an important task since extreme values of the spherical curvature functions will occur at critical points.

TheOREM 2.1. Let $f \in \mathscr{Y}$. Define the level sets

$$
\begin{aligned}
& \lambda_{0}^{r}(f)=\left\{z=r e^{i \theta} \in U: \mathscr{L}_{r}(r, \theta ; f)=0\right\}, \\
& \lambda_{0}^{\theta}(f)=\left\{z=r e^{i \theta} \in U: \mathscr{L}_{\theta}(r, \theta ; f)=0\right\}, \\
& \Lambda_{0}(f)=\left\{z=r e^{i \theta} \in U: \operatorname{Im}\left[z^{2}\{f, z\}\right]=0\right\} .
\end{aligned}
$$

(a) For each $0<r<1$, a point $z=r e^{i \theta} \in \mathscr{C}_{r}$, corresponding to the point $X(r, \theta)$ on the curve $\mathscr{C}_{r}^{\prime \prime}$, is a critical point of the spherical curvature $\kappa(\theta ; r, f)$ if and only if $z=r e^{i \theta} \in \lambda_{0}^{r}(f) \cup \Lambda_{0}(f)$.

(b) For each $-\pi<\theta \leq \pi$, a point $z=r e^{i \theta} \in \mathscr{L}_{\theta}$, corresponding to the point $X(r, \theta)$ on the curve $\mathscr{L}_{\theta}^{\prime \prime}$, is a critical point of the spherical curvature $\kappa(r ; \theta, f)$ if and only if $z=r e^{i \theta} \in \lambda_{0}^{\theta}(f) \cup \Lambda_{0}(f)$.

Proof. (a) By differentiating (1.9), we obtain

$$
\kappa_{\theta}(\theta ; r, f)=\frac{r^{2} \mathscr{L}_{r}(r, \theta ; f) \mathscr{E}_{r \theta}(r, \theta ; f)}{4 \kappa(\theta ; r, f)}
$$

Thus, for a critical point of the spherical curvature $\kappa(\theta ; r, f)$ to occur at a point $z=r e^{i \theta} \in \mathscr{C}_{r}$ corresponding to a point $X(r, \theta) \in \mathscr{C}_{r}^{\prime \prime}$, it is necessary and sufficient that either $\mathscr{L}_{r}(r, \theta ; f)=0$ or $\mathscr{L}_{r \theta}(r, \theta ; f)=0$. (It is possible for both of these quantities to equal zero simultaneously.) If $\mathscr{L}_{r}(r, \theta ; f)=0$, then $\kappa(\theta ; r, f)=1$, an absolute minimum value, and the corresponding $z$ value belongs to the level set $\lambda_{0}^{r}(f)$, and conversely. If $\mathscr{L}_{r \theta}(r, \theta ; f)=0$, then, by (1.10) and (1.11), it must be the case that $\operatorname{Im}\left[z^{2}\{f, z\}\right]=0$, where $z=r e^{i \theta} \in \Lambda_{0}(f)$, and conversely.

(b) By differentiating (1.13), we obtain

$$
\kappa_{r}(r ; \theta, f)=\frac{\mathscr{L}_{\theta}(r, \theta ; f) \mathscr{L}_{r \theta}(r, \theta ; f)}{4 \kappa(r ; \theta, f)} .
$$

Thus, for a critical point of the spherical curvature $\kappa(r ; \theta, f)$ to occur at a point $z=r e^{i \theta} \in \mathscr{L}_{\theta}$ corresponding to a point $X(r, \theta) \in \mathscr{L}_{\theta}^{\prime \prime}$, it is necessary and sufficient that either $\mathscr{E}_{\theta}(r, \theta ; f)=0$ or that $\mathscr{E}_{r \theta}(r, \theta ; f)=0$. (It is possible for both of these quantities to equal zero simultaneously.) If $\mathscr{L}_{\theta}(r, \theta ; f)=0$, then $\kappa(r ; \theta, f)=1$, an absolute minimum value, and the corresponding $z$ value belongs to the level set $\lambda_{0}^{\theta}(f)$, and conversely. If $\mathscr{L}_{r \theta}(r, \theta ; f)=0$, then $\operatorname{Im}\left[z^{2}\{f, z\}\right]=0$, where $z=r e^{i \theta} \in \Lambda_{0}(f)$, and conversely. 
REMARK 2.2. As a consequence of Theorem 2.1, the procedure of determining the critical values of both spherical curvature functions, and subsequently the location of local and absolute minimum and maximum values of either of the spherical curvature functions, is now clear. We first determine the level sets $\lambda_{0}^{r}(f), \lambda_{0}^{\theta}(f)$, and $\Lambda_{0}(f)$. We then compare the values of $\kappa(\theta ; r, f)$ on the set $\left(\lambda_{0}^{r}(f) \cup \Lambda_{0}(f)\right) \cap \mathscr{b}_{r} \subset U$ to each other and the values of $\kappa(r ; \theta, f)$ on the set $\left(\lambda_{0}^{\theta}(f) \cup \Lambda_{0}(f)\right) \cap \mathscr{L}_{\theta} \subset U$ to each other.

Alternately, after all of the critical points have been located, the standard second derivative test may be employed as follows in an attempt to classify them.

For the curves $\mathscr{C}_{r}^{\prime \prime}$, another differentiation of (2.2) with respect to $\theta$ yields

$$
\begin{aligned}
\kappa_{\theta \theta}(\theta ; r, f)= & \frac{r^{2} \mathscr{L}_{r \theta}^{2}(r, \theta ; f)+r^{2} \mathscr{L}_{r}(r, \theta ; f) \mathscr{L}_{r \theta \theta}(r, \theta ; f)}{4 \kappa(\theta ; r, f)} \\
& -\frac{r^{2 \mathscr{L}_{r}}(r, \theta ; f) \mathscr{L}_{r \theta}(r, \theta ; f) \kappa_{\theta}(\theta ; r, f)}{4 \kappa^{2}(\theta ; r, f)} .
\end{aligned}
$$

If the point $X(r, \theta) \in \mathscr{C}_{r}^{\prime \prime}$ corresponds to a critical point for which $\mathscr{L}_{r}(r, \theta ; f)=$ 0 , that is, for which $\kappa(\theta ; r, f)=1$, then the form of the second derivative reduces to

$$
\kappa_{\theta \theta}(\theta ; r, f)=\frac{r^{2 \mathscr{L}_{r \theta}^{2}}(r, \theta ; f)}{4}=\frac{\mathscr{L}^{2}(r, \theta ; f)\left[\operatorname{Im}\left[z^{2}\{f, z\}\right]\right]^{2}}{4}
$$

which is clearly nonnegative at an absolute minimum as expected. On the other hand, if $\mathscr{L}_{r}(r, \theta ; f) \neq 0$ and $\mathscr{L}_{r \theta}(r, \theta ; f)=0$, then the form of the second derivative reduces to

$$
\kappa_{\theta \theta}(\theta ; r, f)=\frac{r^{2} \mathscr{L}_{r}(r, \theta ; f) \mathscr{L}_{r \theta \theta}(r, \theta ; f)}{4 \kappa(\theta ; r, f)}
$$

By differentiating (1.11) with respect to $\theta$, we obtain

$$
r \mathscr{L}_{r \theta \theta}(r, \theta ; f)=\mathscr{L}_{\theta}(r, \theta ; f) \operatorname{Im}\left[z^{2}\{f, z\}\right]+\mathscr{L}(r, \theta ; f) \operatorname{Re}\left[z \frac{d}{d z}\left[z^{2}\{f, z\}\right]\right],
$$

and the second derivative further reduces to the form

$$
\kappa_{\theta \theta}(\theta ; r, f)=\frac{r \mathscr{E}(r, \theta ; f) \mathscr{E}_{r}(r, \theta ; f) \operatorname{Re}\left[z(d / d z)\left[z^{2}\{f, z\}\right]\right]}{4 \kappa(\theta ; r, f)}
$$

clearly indicating the two quantities upon which the classification of the critical point depends. 
For the curves $\mathscr{L}_{\theta}^{\prime \prime}$, another differentiation of (2.3) with respect to $r$ yields

$$
\begin{aligned}
\kappa_{r r}(r ; \theta, f)= & \frac{\mathscr{E}_{r \theta}^{2}(r, \theta ; f)+\mathscr{L}_{\theta}(r, \theta ; f) \mathscr{L}_{r \theta r}(r, \theta ; f)}{4 \kappa(r ; \theta, f)} \\
& -\frac{\mathscr{E}_{\theta}(r, \theta ; f) \mathscr{E}_{r \theta}(r, \theta ; f) \kappa_{r}(r ; \theta, f)}{4 \kappa^{2}(r ; \theta, f)} .
\end{aligned}
$$

If $X(r, \theta) \in \mathscr{L}_{\theta}^{\prime \prime}$ corresponds to a critical point for which $\mathscr{L}_{\theta}(r, \theta ; f)=0$, that is, for which $\kappa(r ; \theta, f)=1$, then the form of the second derivative reduces to

$$
\kappa_{r r}(r ; \theta, f)=\frac{\mathscr{L}_{r \theta}^{2}(r, \theta ; f)}{4}=\frac{\mathscr{L}^{2}(r, \theta ; f)\left[\operatorname{Im}\left[z^{2}\{f, z\}\right]\right]^{2}}{4 r^{2}},
$$

which is clearly nonnegative at an absolute minimum as expected. Now, $X(r, \theta) \in \mathscr{L}_{\theta}^{\prime \prime}$ corresponds to a critical point for which $\mathscr{L}_{\theta}(r, \theta ; f) \neq 0$ and $\mathscr{L}_{r \theta}(r, \theta ; f)=0$, then the form of the second derivative reduces to

$$
\kappa_{r r}(r ; \theta, f)=\frac{\mathscr{L}_{\theta}(r, \theta ; f) \mathscr{E}_{r \theta r}(r, \theta ; f)}{\kappa(r ; \theta, f)} .
$$

By differentiating (1.11) with respect to $r$, we obtain

$$
r \mathscr{L}_{r \theta r}(r, \theta ; f)+\mathscr{L}_{r \theta}(r, \theta ; f)=\frac{\mathscr{L}(r, \theta ; f)}{r} \operatorname{Im}\left[z \frac{d}{d z}\left[z^{2}\{f, z\}\right],\right.
$$

and the second derivative further reduces to the form

$$
\kappa_{r r}(r ; \theta, f)=\frac{\mathscr{L}(r, \theta ; f) \mathscr{L}_{\theta}(r, \theta ; f) \operatorname{Im}\left[z(d / d z)\left[z^{2}\{f, z\}\right]\right]}{4 r^{2} \kappa(r ; \theta, f)}
$$

clearly indicating the two quantities upon which the classification of the critical point depends.

Theoretically, the second derivatives $\kappa_{\theta \theta}(\theta ; r, f)$ and $\kappa_{r r}(r ; \theta, f)$ may also be used to determine the candidates for inflection points of the spherical curvatures $\kappa(\theta ; r, f)$ and $\kappa(r ; \theta, f)$ of the curves $\mathscr{C}_{r}^{\prime \prime}$ and $\mathscr{L}_{\theta}^{\prime \prime}$, respectively, although in practice, this task is computationally formidable.

3. Stationary points of the spherical curvature functions considered as functions of two variables. Although the prescribed spherical curvature functions $\kappa(\theta ; r, f)$ and $\kappa(r ; \theta, f)$ have been defined as a function of one variable (as a function of $\theta$ with $r$ fixed, and vice versa), it is clear that each of them may be considered as a function of two variables on the punctured disk $U-\{0\}$. Upon adopting this point of view, it is natural to ask whether the spherical curvature functions have stationary points in $U-\{0\}$ and how to locate them if they exist. The answer is provided in the following result. 
THEOREM 3.1. Let $f \in \mathscr{Y}$.

(a) Any stationary points of the spherical curvature $\kappa(\theta ; r, f)$, when considered as a function of two variables, belong to the set $\lambda_{0}^{r}(f) \cup\left(\Lambda_{0}(f) \cap \Xi_{0}^{+}(f)\right)$, where $\Xi_{0}^{+}(f)$ is defined within the proof. Furthermore, if the stationary point $z \in \Lambda_{0}(f) \cap \Xi_{0}^{+}(f)$, then it is necessary that $z^{2}\{f, z\}$ be real and greater than $1 / 2$ at that point.

(b) Any stationary points of the spherical curvature $\kappa(r ; \theta, f)$, when considered as a function of two variables, belong to the set $\lambda_{0}^{\theta}(f) \cup\left(\Lambda_{0}(f) \cap \Xi_{0}^{-}(f)\right)$, where $\Xi_{0}^{-}(f)$ is defined within the proof. Furthermore, if the stationary point $z \in \Lambda_{0}(f) \cap \Xi_{0}^{-}(f)$, then it is necessary that $z^{2}\{f, z\}$ be real and less than $1 / 2$ at that point.

Proof. (a) Since

$$
\begin{aligned}
& \kappa_{r}(\theta ; r, f)=\frac{r^{2} \mathscr{E}_{r}(r, \theta ; f)\left[\mathscr{E}_{r r}(r, \theta ; f)+(1 / r) \mathscr{E}_{r}(r, \theta ; f)\right]}{4 \kappa(\theta ; r, f)}, \\
& \kappa_{\theta}(\theta ; r, f)=\frac{r^{2} \mathscr{E}_{r}(r, \theta ; f) \mathscr{E}_{r \theta}(r, \theta ; f)}{4 \kappa(\theta ; r, f)}
\end{aligned}
$$

it is clear that both derivatives will vanish if $\mathscr{L}_{r}(r, \theta ; f)=0$, that is, if $z=$ $r e^{i \theta} \in \lambda_{0}^{r}(f)$. In this case, $\kappa(\theta ; r, f)=1$ which corresponds to an absolute minimum value. On the other hand, a stationary point also occurs at a point $z=r e^{i \theta} \in U$ whenever $\mathscr{L}_{r \theta}(r, \theta ; f)=0$ and $\mathscr{L}_{r r}(r, \theta ; f)+(1 / r) \mathscr{L}_{r}(r, \theta ; f)=0$. But $\mathscr{L}_{r \theta}(r, \theta ; f)=0$ if and only if $\operatorname{Im}\left[z^{2}\{f, z\}\right]=0$ by (1.11). In this case, $z \in$ $\Lambda_{0}(f)$. Also, $\mathscr{L}_{r r}(r, \theta ; f)+(1 / r) \mathscr{L}_{r}(r, \theta ; f)=0$ if and only if

$$
\frac{2}{\mathscr{L}^{2}(r, \theta ; f)}\left(1+|\Phi(z ; f)|^{2}\right)=\operatorname{Re}\left[z^{2}\{f, z\}\right]-\frac{1}{2}
$$

by (1.20). Letting $\Xi_{0}^{+}(f)$ denote the set of $z \in U$ for which this equation holds, the first assertion of part (a) is established. If a stationary point $z \in \Lambda_{0}(f) \cap$ $\Xi_{0}^{+}(f)$, then it is necessary that $z^{2}\{f, z\}$ be real and greater than $1 / 2$ at that point.

(b) Since

$$
\begin{aligned}
& \kappa_{r}(r ; \theta, f)=\frac{\mathscr{E}_{\theta}(r, \theta ; f) \mathscr{E}_{r \theta}(r, \theta ; f)}{4 \kappa(r ; \theta, f)}, \\
& \kappa_{\theta}(r ; \theta, f)=\frac{\mathscr{E}_{\theta}(r, \theta ; f) \mathscr{E}_{\theta \theta}(r, \theta ; f)}{4 \kappa(r ; \theta, f)},
\end{aligned}
$$

it is clear that both derivatives will vanish if $\mathscr{L}_{\theta}(r, \theta ; f)=0$, that is, if $z \in \lambda_{0}^{\theta}(f)$. In this case, $\kappa(r ; \theta, f)=1$ which corresponds to an absolute minimum value. On the other hand, a stationary point also occurs at a point $z=r e^{i \theta} \in U$ whenever $\mathscr{L}_{r \theta}(r, \theta ; f)=0$ and $\mathscr{L}_{\theta \theta}(r, \theta ; f)=0$. Again, $\mathscr{L}_{r \theta}(r, \theta ; f)=0$ if and only if $\operatorname{Im}\left[z^{2}\{f, z\}\right]=0$ by (1.11). In this case, $z \in \Lambda_{0}(f)$. Also, $\mathscr{L}_{\theta \theta}(r, \theta ; f)=0$ 
if and only if

$$
\frac{2}{\mathscr{L}^{2}(r, \theta ; f)}\left(1+|\Phi(z ; f)|^{2}\right)=\frac{1}{2}-\operatorname{Re}\left[z^{2}\{f, z\}\right]
$$

by (1.21). Letting $\Xi_{0}^{-}(f)$ denote the set of $z \in U$ for which this equation holds, the first assertion of part (b) is established. If a stationary point $z \in \Lambda_{0}(f) \cap$ $\Xi_{0}^{-}(f)$, then it is necessary that $z^{2}\{f, z\}$ be real and less than $1 / 2$ at that point.

We conclude from Theorem 3.1 that no point $z \in \Lambda_{0}(f)$ can simultaneously be a stationary point for both spherical curvature functions $\kappa(\theta ; r, f)$ and $\kappa(r ; \theta, f)$.

Having located the stationary points of both spherical curvature functions, the standard Hessian test may be used to classify them when the test is not inconclusive.

4. Subharmonicity and maximum principles for spherical curvature. It is natural to ask whether there exist maximum principles for the spherical curvature functions on subdomains of $U-\{0\}$. It is well known that subharmonic functions satisfy principles of this type. Hence, we attempt to discover conditions under which we may conclude that powers of the spherical curvature functions are subharmonic.

Let $D$ be a simply connected domain with piecewise smooth boundary $\partial D$ contained in $U-\{0\}$. (Of course, more general domains may be considered.) Note that both spherical curvature functions given by (1.9) and (1.13) have the algebraic form $w=\left(1+u^{2}\right)^{1 / 2}$, where either $u=-(1 / 2) r \mathscr{L}_{r}(r, \theta ; f)$ or $u=+(1 / 2) \mathscr{L}_{\theta}(r, \theta ; f)$. A short computation shows that

$$
\Delta w^{\alpha}=\alpha\left(1+u^{2}\right)^{\alpha / 2}\left[\left(1+u^{2}\right) u \Delta u+\left((\alpha-1) u^{2}+1\right)\left(u_{r}^{2}+\frac{1}{r^{2}} u_{\theta}^{2}\right)\right]
$$

Since the function $u \Delta u$ is continuous on $\bar{D}$, it must have an absolute minimum there, which may be negative. For $w^{\alpha}$ to be subharmonic, the other quantity within the square brackets must compensate for the possible negativity. This will occur provided that

$$
\min _{\bar{D}} u^{2}>0
$$

that

$$
\min _{\bar{D}}\left(u_{r}^{2}+\frac{1}{r^{2}} u_{\theta}^{2}\right)>0,
$$


and that $\alpha$ is sufficiently large. On the other hand, if $u \Delta u$ is positive, then the choice $\alpha=1$ implies that $w$ is subharmonic.

THEOREM 4.1. Let $f \in \mathscr{S}$ and let $D$ be a simply connected domain with piecewise smooth boundary $\partial D$ contained in $U-\{0\}$.

(a) If $\bar{D}$ does not meet either $\lambda_{0}^{r}(f)$ or $\Lambda_{0}(f)$, then there exists $\hat{\alpha} \geq 1$ such that $\kappa^{\alpha}(\theta ; r, f)$ is subharmonic on $D$ for all $\alpha \geq \hat{\alpha}$.

(b) If $\bar{D}$ does not meet either $\lambda_{0}^{\theta}(f)$ or $\Lambda_{0}(f)$, then there exists $\hat{\beta} \geq 1$ such that $\kappa^{\beta}(r ; \theta, f)$ is subharmonic on $D$ for all $\beta \geq \hat{\beta}$.

Consequently, under the given conditions, the maximum values of $\kappa^{\alpha}(\theta ; r, f)$ and $\kappa^{\beta}(r ; \theta, f)$, when considered as functions of two variables, must occur on the boundary of the domain $D$.

Proof. (a) Let $u=-(1 / 2) r_{\mathscr{E}_{r}}(r, \theta ; f)$. Then (4.2) holds provided that $\bar{D}$ does not meet $\lambda_{0}^{r}(f)$ and (4.3) holds provided that $\bar{D}$ does not meet $\Lambda_{0}(f)$, for $\left(u_{r}^{2}+\left(1 / r^{2}\right) u_{\theta}^{2}\right) \geq(1 / 4) \mathscr{E}_{r \theta}^{2}>0$ on $\bar{D}$ due to relation (1.11). Thus, the stated conditions imply that the Laplacian $\Delta \kappa^{\alpha}(\theta ; r, f)$ will be positive if $\alpha$ is large enough. An appeal to the maximum principle for subharmonic functions now allows us to conclude that $\kappa^{\alpha}(\theta ; r, f)$ attains its maximum value on the boundary of $D$.

(b) The proof of part (b) is similar to the proof of part (a) if we substitute $u=+(1 / 2) \mathscr{E}_{\theta}(r, \theta ; f)$.

COROLLARY 4.2 (maximum principles for the spherical curvature functions). Let $f \in \mathscr{S}$ and let $D$ be a simply connected domain with smooth boundary $\partial D$ contained in $U-\{0\}$. Then, under the conditions stated in Theorem 4.1, the maximum values of $\kappa(\theta ; r, f)$ and $\kappa(r ; \theta, f)$, considered as functions of two variables, must occur on the boundary of $D$.

Proof. Choose $\alpha>1$ (resp., $\beta>1$ ) large enough so that $\kappa^{\alpha}(\theta ; r, f) \geq 1$ (resp., $\kappa^{\beta}(r ; \theta, f) \geq 1$ ) is subharmonic on $D$. Since its maximum value occurs on the boundary of $D$, we need only to extract the $\alpha$ th (resp., $\beta$ th) root to obtain the result. Note that $\kappa(\theta ; r, f)$ (resp., $\kappa(r ; \theta, f))$ is itself subharmonic if $\hat{\alpha}<1$ (resp., $\hat{\beta}<1$ ).

5. Topological properties of the level sets $\lambda_{0}^{r}(f), \lambda_{0}^{\theta}(f)$, and $\Lambda_{0}(f)$. The importance of the level sets $\lambda_{0}^{r}(f), \lambda_{0}^{\theta}(f)$, and $\Lambda_{0}(f)$ has been firmly established in Theorems 2.1, 3.1, and 4.1. Therefore, in view of our central purpose, it is important to discuss these sets in further detail.

In view of the representations (1.17) and (1.18), it is clear that the sets $\lambda_{0}^{r}(f)$ and $\lambda_{0}^{\theta}(f)$ are the level sets of infinitely smooth surfaces for every $f \in \mathscr{Y}$. Some additional topological properties of these level sets are established here.

Proposition 5.1. Let $f \in \mathscr{S}$.

(a) The level set $\lambda_{0}^{r}(f)$ does not contain an open subset of $U$.

(b) The level set $\lambda_{0}^{\theta}(f)$ contains an open subset of $U$ if and only if $f(z)=z$, in which case $\lambda_{0}^{\theta}(f)=U$. 
(c) The level sets $\lambda_{0}^{r}(f)$ and $\lambda_{0}^{\theta}(f)$ do not contain Jordan curves (i.e., simple closed curves) which bound simply connected subdomains of $U-\{0\}$.

Proof. (a) Recall (1.17). The numerator of $\operatorname{Re} \Phi(z ; f)$ is the difference of two functions: the first $N_{1}(z)$ of which is harmonic and the other $N_{2}(z)$ which is not. They can be equal on an open set if and only if they are both constant. The function $N_{1}(z)$ is constant on an open set if and only if $f(z)=z$, but in this eventuality, $N_{2}(z)$ is a nonconstant function of $r$.

(b) Recall (1.18). The argument for $\lambda_{0}^{\theta}(f)$ proceeds in a similar manner. If we choose $f(z)=z$, then $f^{\sharp}(\rho, \theta)=1 /\left(1+\rho^{2}\right)$ and $\mathscr{L}_{\theta}(\rho, \theta ; f) \equiv 0$. Thus, for all values of $\rho$, we have $\kappa(\rho ; \theta, f) \equiv 1$ so that $\lambda_{0}^{\theta}(f)=U$.

(c) Suppose, on the contrary, that the level set $\lambda_{0}^{r}(f)$ contains a Jordan curve $\gamma$ which bounds a simply connected subdomain $G$ of $U-\{0\}$. Since $\kappa(\theta ; r, f) \geq$ 1 , considered as a function of two variables, is nonconstant and continuous on $\bar{G}$ and $\kappa(\theta ; r, f)=1$ on $\gamma=\partial G$, it has a maximum value in the interior of $G$ at some point $z_{0}=r_{0} e^{i \theta_{0}}$, and we have $\kappa\left(\theta_{0} ; r_{0}, f\right) \geq \kappa(\theta ; r, f)>1$ throughout $G$.

Now, let $\alpha$ be an analytic, univalent mapping of $G$ onto the unit disk $U$. Let $\gamma_{r}=\alpha^{-1}(|z|=r)$ and let $G_{r}$ denote the interior of $\gamma_{r}$. For $\epsilon>0$ small, there exists an $r_{\epsilon}$ such that $\left|\mathscr{L}_{r}(r, \theta ; f)\right|<\epsilon$ on $\gamma_{r_{\epsilon}}$ since $\mathscr{L}_{r}(r, \theta ; f)$ is continuous on $\bar{G}$ and $\mathscr{L}_{r}(r, \theta ; f)=0$ on $\gamma$. It follows from this inequality and (1.9) that $\kappa(\theta ; r, f)<\left(1+(1 / 4) \epsilon^{2}\right)^{1 / 2}$ on $\gamma_{r_{\epsilon}}$. This is a contradiction to Corollary 4.2 , the maximum principle for the spherical curvature for if $\epsilon$ is small enough, $z_{0} \in G_{r}$ and $\left(1+(1 / 4) \epsilon^{2}\right)^{1 / 2}<\kappa\left(\theta_{0} ; r_{0}, f\right)$.

The argument for $\lambda_{0}^{\theta}(f)$ proceeds in a similar manner.

REMARK 5.2. In view of Proposition 5.1, it is natural to ask whether the level set $\lambda_{0}^{r}(f)$ may be empty. The answer is affirmative. For if $f(z)=z$, then $\mathscr{L}(\rho, \theta ; f)=\left(\rho^{2}+1\right) / \rho$ and $\mathscr{L}_{r}(\rho, \theta ; f)=1-1 / \rho^{2}<0$ for all $0<\rho<1$ so that $\lambda_{0}^{r}(f)=\varnothing$.

If $f(z)=z+a_{2} z^{2}+a_{3} z^{3}+\cdots$, then the asymptotic expansion

$$
\kappa(\theta ; \rho, f)=\frac{1}{2 \rho}+\frac{1}{2}\left(1-\left|a_{2}\right|^{2}-3 \operatorname{Re}\left\{\left(a_{2}^{2}-a_{3}\right) e^{2 i \theta}\right\}\right) \rho+\cdots
$$

clearly shows that $\kappa(\theta ; \rho, f)>1$ so that $\mathscr{L}_{r}(\rho, \theta ; f) \neq 0$, for small values of $\rho$, implying that the level set $\lambda_{0}^{r}(f)$ does not get too close to the origin.

Indeed, for every $r \in(0, \tilde{r})$ and every $\theta \in(-\pi,+\pi]$, we have

$$
\min _{f \in \mathscr{S}} \kappa(\theta ; r, f)>1
$$

where $\tilde{r}=0.19783 \ldots$ is the only root of the equation

$$
1-8 r+22 r^{2}-40 r^{3}+22 r^{4}-8 r^{5}+r^{6}=0
$$


in the interval $(0,1)$. To see this, one needs only to apply the familiar estimates given in [2, pages 32-35] to each part of the formula for $\mathscr{L}_{r}(\rho, \theta ; f)$ given by (1.17).

However, for larger values of $\rho, \mathscr{L}_{r}(\rho, \theta ; f)$ may equal 0 . Indeed, for every $r \in[\hat{r}, 1)$ and every $\theta \in(-\pi,+\pi]$, we have

$$
\min _{f \in \mathscr{S}} \kappa(\theta ; r, f)=1
$$

where $\hat{r}=0.25971 \ldots$ is the only root of the equation

$$
1-10 r^{2}-16 r^{3}-10 r^{4}+r^{6}=0
$$

in the interval $(0,1)$. There is an infinite number of functions for which this minimum is attained. This result has been previously established in [3, page 107]. Thus, for these larger values of $\rho$, there may exist functions $f \in \mathscr{S}$ for which $\lambda_{0}^{r}(f) \neq \varnothing$.

REMARK 5.3. It is equally important to discuss the level set $\Lambda_{0}(f)$. Since $\operatorname{Im}\left[z^{2}\{f, z\}\right]$ is a harmonic function, some of the properties of the level set $\Lambda_{0}(f)$ follow from well-known properties of harmonic functions. For instance, $\Lambda_{0}(f)$ will contain neither an isolated point nor an arc which terminates at an interior point of $U$ since either of these occurrences would violate the mean value property for harmonic functions. Also, if the level set $\Lambda_{0}(f)$ is to contain either an open subset of $U$ or a closed Jordan curve, then $\operatorname{Im}\left[z^{2}\{f, z\}\right]$ would be identically zero on $U$, due to an application of the maximum principle for harmonic functions. This possibility could occur if, for instance, $f(z)=z /(1-$ $c z$ ) for some constant $c$.

\section{REFERENCES}

[1] S. D. Bernardi, Bibliography of Schlicht Functions, Mariner Publishing, Tampa, 1982.

[2] P. L. Duren, Univalent Functions, Grundlehren der Mathematischen Wissenschaften, vol. 259, Springer-Verlag, New York, 1983.

[3] S. M. Zemyan, On the extremal curvature and torsion of stereographically projected analytic curves, Tamkang J. Math. 28 (1997), no. 2, 101-117.

Stephen M. Zemyan: Department of Mathematics, The Pennsylvania State University, Mont Alto, PA 17237-9799, USA

E-mail address: smz3@psu.edu 


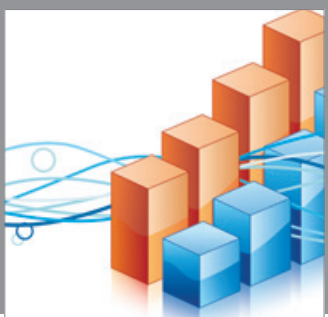

Advances in

Operations Research

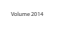

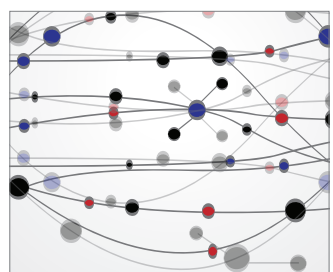

\section{The Scientific} World Journal
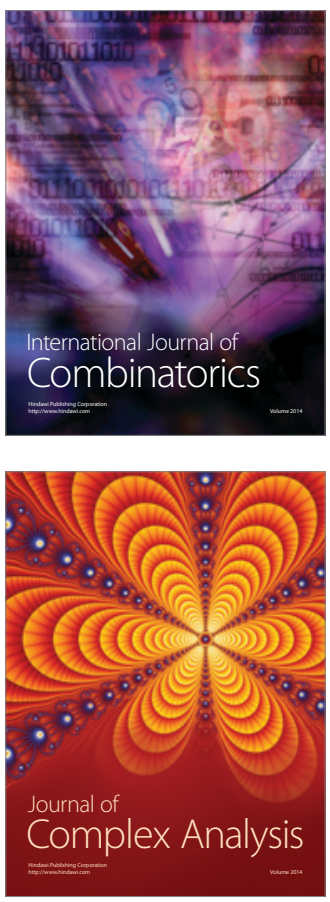

International Journal of

Mathematics and

Mathematical

Sciences
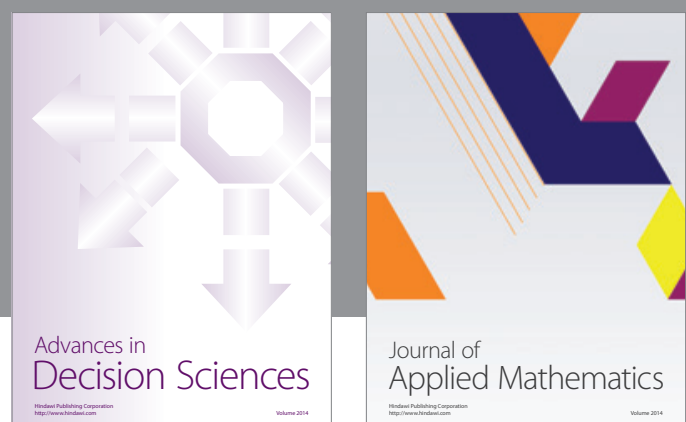

Journal of

Applied Mathematics
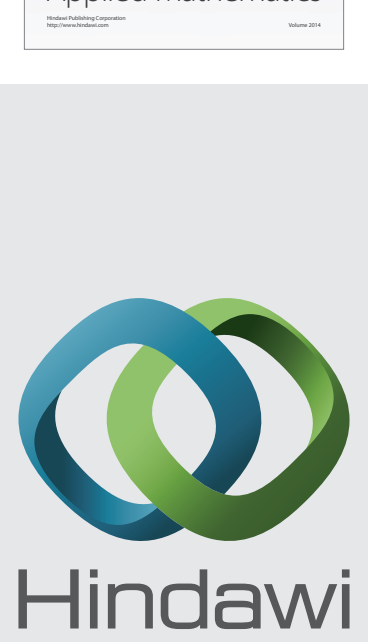

Submit your manuscripts at http://www.hindawi.com
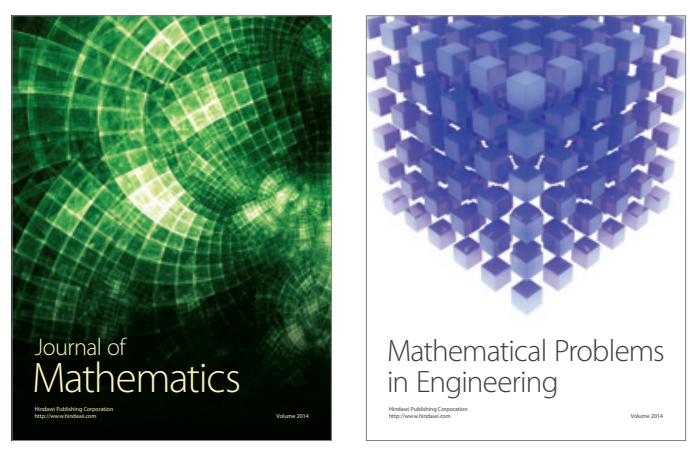

Mathematical Problems in Engineering
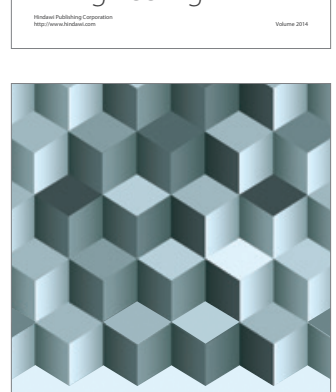

Journal of

Function Spaces
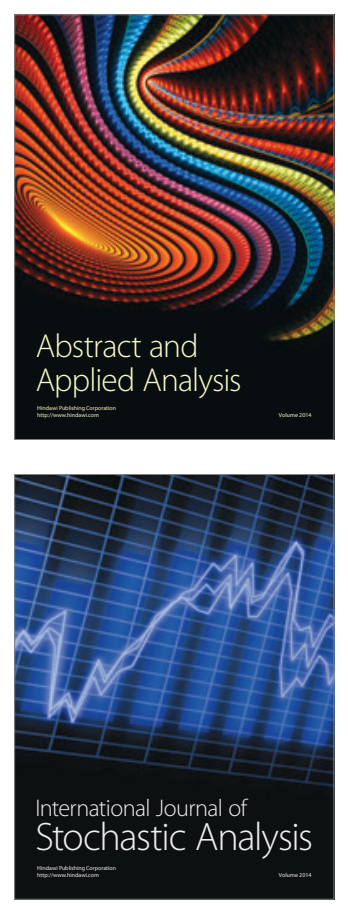

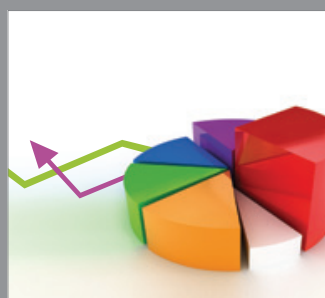

ournal of

Probability and Statistics

Promensencen
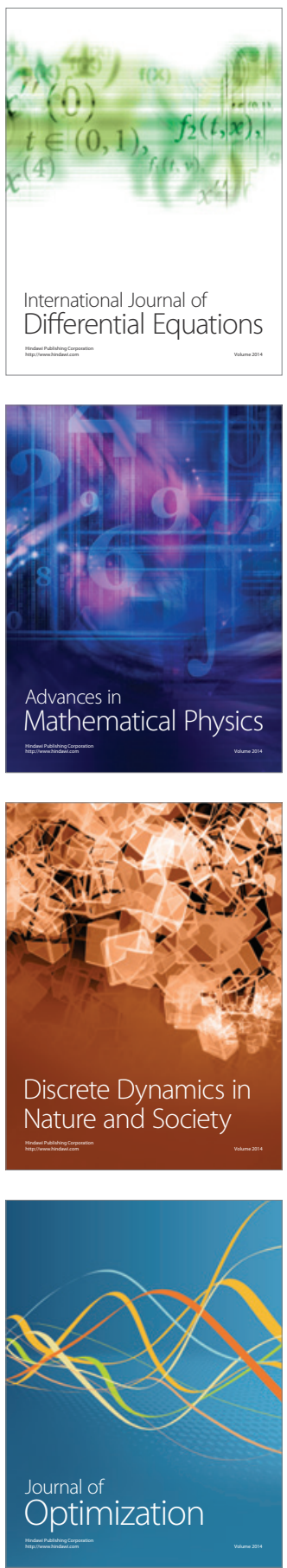